\title{
Standard Operating Procedure for the Microscopical Analysis of Foreign Object Debris (FOD)
}

\author{
Brown, Richard S. ${ }^{1}$ \\ 1. MVA Scientific Consultants, Duluth, GA, USA.
}

The microscopical analysis of unknown particles or foreign object debris (FOD) requires the flexibility to modify the analysis procedure during the analysis. This type of analysis procedure challenges the classic standard operating procedure (SOP) that requires predetermined testing to be followed to obtain a consistent level of quality and a reliable analytical result. Unfortunately, there is not an SOP for the "analysis of all things" which is precisely the type of analysis the investigative microscopist performs on a daily basis. Any analysis procedure when applied to FOD that occurs in products or processes requires flexibility because the types of particles found determine the analysis procedure. This procedure has been developed over the last 20 years and continues to change as new instrumentation is added.

The initial analysis is performed by gross inspection of the FOD or the object with the adhering FOD. Low magnification examination using a stereomicroscope is performed next in order for the microscopist to decide upon an analysis procedure. Hard, shiny, opaque particles are removed and analyzed using scanning electron microscopy-energy dispersive x-ray spectrometry (SEM-EDS). Occasionally, FOD is observed embedded in an object. This situation requires either the entire object to be examined in the SEM or the object is sectioned to accommodate analysis by SEM-EDS. Both sample preparation techniques are employed when examining engine components. Soft, elastomeric or plastic transparent particles are subsamples for analysis by polarized light microscopy (PLM) and Fourier transform infrared microspectroscopy (FTIR). SEM-EDS analysis is also performed to characterize mineral fillers and pigments that may be present. Confocal Raman microscopy and analytical electron microscopy (AEM) can also be used for smaller particles. For small particles (less than 100 micrometers) the order in which the analyses are performed is carefully selected to obtain the maximum amount of information before the particle is exhausted by the analysis. SEM-EDS can be performed on a small particle using a beryllium planchet as a substrate, then the particle can be transferred to a stainless steel substrate for confocal Raman microscopy or FTIR using an ATR objective. Finally, the particle can be mounted between a slide and coverslip for PLM analysis. Particles that are larger or that are aggregates of smaller particles, can be subsampled for analysis using multiple techniques simultaneously.

By using multiple microscopical analysis techniques on the same sample the results of each analysis can be compared to determine the composition of the sample. Additionally, results obtained from multiple techniques will provide a quality control check on the overall analysis protocol. For instance, if fluoropolymer fragments or shavings are present as FOD, SEM-EDS should detect carbon and fluorine, PLM should find particles with optical properties consistent with fluorocarbon polymers and FTIR should provide spectral data that is consistent with a fluorocarbon polymer. In the case of aggregates or polymeric materials containing mineral fillers and pigments, data obtained from each technique should be complimentary. Finding data that is not complimentary will require additional analysis using a different technique or the repeat of a previous technique. Particles containing multiple layers of materials, mineral fillers, dyes or multiple phases will require special separation techniques such as low temperature oxygen plasma ashing, ion mill cross section polishing, sublimation, solvent extraction or pyrolysis. 
Complimentary microscopical techniques provide a rapid, reliable, consistent and quality approach to the characterization and identification of FOD. Cross-checks between techniques provide reliable data and the flexibility to respond to the investigative analysis of unknown particles.

\section{References:}

[1] Barrette, Roger W., Hyde, Adam M., and Brown, Richard S., "Investigation of Hit and Run Crashes", Chapter 14, Traffic Crash Investigation, $11^{\text {th }}$ Edition, Fricke, Lynn B. and Baker, J. Stannard, Ed. , Northwestern University for Public Safety, 2014.

[2] Brown, R.S., "Characterization and Source Identification of Fugitive Dusts by Light and Electron Microscopy", Proceedings of SPIE, Scanning Microscopies 2011: Advanced Microscopy Technologies for Defense, Homeland Security, Forensic, Life Environmental and Industrial Sciences, Vol. 8036 803607.

[3] Millette, J.R. and Brown, R.S., Environmental Forensic Microscopy. Chapter 13 In: Introduction to Environmental Forensics, $2^{\text {nd }}$ Ed., B. L. Murphy and R. D. Morrison, Eds., Academic Press, Elsevier, Amsterdam. pp. 611-635, 2007 